\author{
Military Technical College \\ Kobry El-Kobbah, \\ Cairo, Egypt
}

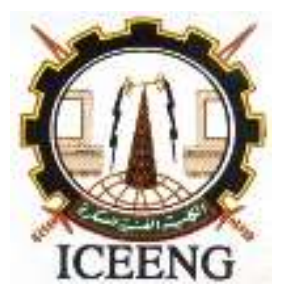

\author{
$11^{\text {th }}$ International Conference \\ on Electrical Engineering \\ ICEENG 2018
}

\title{
Performance Evaluation and Analysis of Very High-Resolution Satellite Telescopes
}

Walid A*. Attia, Taher M. Bazan*, Fawzy Eltohamy* and Mahmoud Fathy**

\begin{abstract}
In this paper, a system performance evaluation of very high-resolution satellite telescopes is investigated. The proposed system is based on Korsch optical design and employing a time-delay and integration charge coupled device (TDI-CCD) detector to achieve a ground sampling distance (GSD) of $25 \mathrm{~cm}$. The key performance metrics considered in this investigation are the absolute and differential signal-to-noise ratio (SNR) and the total modulation transfer function (MTF) of the system. The system MTF has been computed including diffraction, aberration, optical manufacturing, smear and detector as the main contributors for evaluation the MTF. The SNR has been analyzed at different target albedos, sun and sensor angles. In addition, the spatial resolution in terms of cut-off resolution for minimum and maximum illumination conditions is assessed. The analysis shows that the predicted ground resolutions for minimum and maximum illumination situations are 26.4 and $43 \mathrm{~cm}$, respectively
\end{abstract}

\section{KEY WORDS}

Performance evaluation, Modulation transfer function, Signal-to-noise ratio.

\section{Introduction}

System performance evaluation is an essential stage in the design of high-resolution satellite telescopes prior to development process. This paper discusses the main metrics used to evaluate the performance of the remote sensing satellite's electrooptical imaging system at the design phase. These metrics are the modulation transfer function (MTF), signal to noise ratio (SNR) and the linear ground resolution [1]. Most studies have focused on the estimation of absolute SNR but a very few research has used a differential SNR as another key metric for image quality requirement. However, this metric was used for $1 \mathrm{~m}$ resolution of Kazakhstan satellite developed by the European Aeronautic Defense and Space Company (EADS) Astrium [2].

* Egyptian Armed Forces.

** Benha University 
In this paper the system under study is a proposed electro-optical imaging system consisting of a Korsch optical scheme with a ground sampling distance (GSD) of 0.25 $\mathrm{m}$ and a typical linear TDI-CCD detector operating in the visible spectrum band [3]. The proposed telescope is supposed to be mounted on a satellite at a low earth orbit. The technical characteristics of the imaging system are summarized in Table I. Performance evaluation and analysis of the proposed very high-resolution satellite telescope using all the traditional metrics as well as the differential SNR is investigated.

Table 1: The main parameters of a very high-resolution satellite telescope

\begin{tabular}{|c|c|c|}
\hline Parameter name & symbol & Value \\
\hline Orbit altitude & $H$ & $500 \mathrm{Km}$ \\
\hline Orbit inclination & $i$ & $98.5^{0}$ sun synchronous \\
\hline Pixel size & $\Delta$ & $10 \mu \mathrm{m}$ \\
\hline Number of TDI steps & $N_{T D I}$ & $8,16,32,64$ and128 controllable \\
\hline Full well capacity & $F W C$ & $250 \mathrm{Ke}-$ \\
\hline Average quantum efficiency & $q$ & $50 \%$ \\
\hline Dark noise & $\sigma_{d}$ & $100 \mathrm{e}-$ \\
\hline Line frequency & $L_{f}$ & Up to $30 \mathrm{KHz}$ \\
\hline Bit resolution & $n$ & 12 bit \\
\hline Aperture diameter & $D_{a p}$ & $1.401 \mathrm{~m}$ \\
\hline Obscured diameter & $D_{o b}$ & $0.331 \mathrm{~m}$ \\
\hline Focal length & $f$ & $20 \mathrm{~m}$ \\
\hline Optical transmittance & $\tau_{\mathrm{op}}$ & $90 \%$ \\
\hline Meteorological visibility & $M_{\mathrm{v}}$ & $23 \mathrm{~km}$ \\
\hline Atmospheric model & & Mid-latitude summer \\
\hline
\end{tabular}

The paper is organized as follows. Section 2 is devoted to estimate the total system MTF based on optical, detector, atmospheric, and smear issues. The analysis of SNR according to particular illumination scenarios is investigated in details in Section 3. The linear ground resolution is assessed in Section 4. Finally, the main conclusions are summarized in Section 5.

\section{MTF Estimation}

The MTF is a vital parameter for performance evaluation of any electro-optical imaging system. MTF is defined as the spatial frequency response of the imaging system as it shows the capability of the imaging system to reproduce an image of a sinusoidal wave target in the scene at different spatial frequencies. The total MTF of the system is the product of the MTFs of each component that contribute of producing the final image. The main contributors are the optics, the detector, the smear and the atmosphere. The total MTF of the system is defined by [4]:

$$
M T F_{\text {tot }}=M T F_{\text {optics }} \times M T F_{\text {detector }} \times M T F_{\text {smear }} \times M T F_{\text {atmosphere }}
$$

The optical system is designed to be diffraction limited. However, in reality, the diffraction limited systems cannot be achieved due to the imperfections of manufacturing and alignment. So a tolerance MTF is considered to reflect this effect. In this study, the optical MTF is considered as the product of the diffraction limited and 
the tolerance MTFs [3]. The CCD photodetector is a spatial system, the MTF due to spatial averaging on the detector aperture is determined by [5]:

$$
M T F_{\text {detector }}(v)=\frac{\sin (\pi \Delta v)}{(\pi \Delta v)}
$$

where $\Delta$ is the detector pixel size and $v$ is the spatial frequency in $(\mathrm{Lp} / \mathrm{mm})$. Smear is defined by line of sight linear shift during the exposure time. For Linear TDI photodetectors, the exposure time $\left(t_{\text {exp }}\right)$ is limited by the number of TDI lines $\left(N_{T D I}\right)$ and the line integration time $\left(t_{\text {int }}\right)$. In general, the longer the exposure time, the higher the smear. However, there are many sources of smear [6]. In this study, the smear is estimated using the model proposed in [7] where the smear is defined as the vector sum of the velocity mismatch between the target rate and the electronic line frequency at different TDI lines for two acquisition scenarios

The atmospheric MTF is a combination of turbulence and aerosol effects. The atmospheric turbulence occurs due to the wavefront distortion of light during propagation through a long path of atmosphere whereas the aerosol MTF arises due to the scattering and absorption by molecules in the atmosphere. However, the atmospheric MTF is modelled here taking in consideration the turbulence effect as the primary source [4]. The total MTF of the system is generally estimated in terms of the spatial frequency normalized to detector sampling frequency $\left(v / v_{\text {samp }}\right)$. The detector sampling frequency $\left(v_{\text {samp }}\right)$ is determined by $(1 / p)$ where $p$ is the pixel pitch. The Nyquist frequency $\left(v_{n y q}\right)$ is the highest spatial frequency in which the detector can respond to. Nevertheless, it is determined by $(1 / 2 p)$ which corresponds to the normalized spatial frequency of 0.5 . The total MTF of the system has been estimated using different TDI lines at two acquisition scenarios of observation angle $(\theta)$. The total MTF for the first scenario where $\theta=0^{\circ} \quad$ (i.e., at nadir) and $N_{T D I}=8$ whereas for the second one $\theta=$ $36.5^{\circ}$ (off-nadir) and $N_{T D I}=64$ as illustrated in Fig. $1 \mathrm{a}$ and $1 \mathrm{~b}$, respectively. It is clear that the total MTFs at the Nyquist frequency for the two scenarios are 0.19 and 0.14 , respectively.

\section{Analysis of SNR}

The SNR is an important metric used to evaluate the radiometric performance of the electro-optical imaging system. The SNR is defined as the mean signal to the standard deviation of the total noise. Therefore, it is required to calculate both the signal and noise. The signal from target $\left(S_{t a r}\right)$ is considered as the number of photoelectrons generated in proportional to the sun irradiance falling on the photodetector. The output signal is calculated by [8]:

$$
S_{\text {tar }}=\frac{A_{d e t} \pi(1-\epsilon) t_{\text {exp }}}{4(f \#)^{2} h c} \int_{\lambda_{\min }}^{\lambda_{\max }} L_{t a r}(\lambda) q(\lambda) \tau_{o p}(\lambda) \lambda d \lambda
$$

where $A_{\text {det }}$ is the detector area, $h$ is the plank's constant, $c$ is the speed of light, $f \#$ is the F-number and $f$ is the focal length of the optical system. $\epsilon$ is the obscuration coefficient defined as the ratio of the clear aperture area to the obscured one, i.e., $\left(D^{2} a p\right.$ $\left./ D^{2}{ }_{o b}\right)$. $L_{t a r}$ is the target radiance which is the sum of the direct and diffuse radiances ( $\left.L_{\text {dir }}+L_{\text {dif }}\right) . L_{\text {dir }}$ is the direct radiance defined by the radiance of the direct beam from the sun reflected on the target toward the sensor and $L_{\text {dif }}$ is the diffuse radiance that is the 
down-welled scattered sun radiance reflected on the target toward the sensor. $q(\lambda)$ is the spectral quantum efficiency and $\tau_{o p}(\lambda)$ is the spectral transmittance of the optical system.

Among all noise sources, only the main contributors will be taken into consideration; the photon noise $\left(\sigma_{p h}\right)$, quantization noise $\left(\sigma_{q u}\right)$ and the dark noise $\left(\sigma_{d}\right)$. However, the standard deviation of the total noise is the square root of the sum of the noise components variances expressed by:

$$
\sigma_{t o t}=\sqrt{\sigma_{p h}^{2}+\sigma_{q u}^{2}+\sigma_{d}^{2}}
$$

where

$$
\sigma_{p h}=\sqrt{S_{t a r}+S_{b k g}}
$$

$S_{b k g}$ is the background signal due to haze radiance $\left(L_{h a z e}\right)$. The quantization noise arises from the process of analog to digital converter and can be given by [8]:

$$
\sigma_{q u}=\frac{Q S E}{\sqrt{12}}=\frac{F W C}{2^{n} \sqrt{12}}
$$

where QSE is the quantization step equivalence, $F W C$ is the pixel full well capacity and $n$ is the analog to digital converter bit resolution.

\subsection{Absolute SNR}

The absolute signal to noise ratio $\left(S N R_{\rho}\right)$ is defined by considering the target under observation as a uniform background with constant albedo $(\rho)$ [8]:

$$
S N R_{\rho}=\frac{S_{\text {tar }}}{\sigma_{\text {tot }}} \mid \rho
$$

\subsection{Radiometric calculation}

Calculation of the different radiance components reaching the sensor through the atmosphere is complex. Thus, the software programs like MOSART and MODTRAN are usually used to calculate the top of atmosphere (TOA) radiances using standard atmospheric and aerosol models. TOA radiances at different combinations of sun and observation angles were estimated using the MOSART taking in consideration the following initial assumptions [9]:

1) The surface reflectance is Lambertain and the target albedo assigned to be 1 .

2) $q(\lambda)$ and $\tau_{o p}(\lambda)$ are counted as constant over the spectral band.

3) The atmospheric model is Mid-Latitude summer and the meteorological visibility range is $23 \mathrm{Km}$.

4) The operating spectral operating band is $0.4-0.9 \mu \mathrm{m}$.

Direct and diffuse radiances are calculated first at $\rho=1$. Therefore, the target radiance at any other albedo is simply the multiplication of the target radiance by the required albedo value. After some manipulations, Eq. (3) can be rewritten as: 


$$
S_{\text {tar } \mid \rho}=\frac{\left.\rho L_{t a r}\right|_{\rho=1} A_{\text {det }} \pi(1-\epsilon) N_{T D I} t_{\text {int }} q \tau_{\text {opt }}}{4(f \#)^{2}}
$$

Therefore, the background signal can be expressed as:

$$
S_{b k g}=\frac{L_{\text {haze }} A_{d e t} \pi(1-\epsilon) N_{T D I} t_{i n t} q \tau_{o p t}}{4(f \#)^{2}}
$$

The integration time in Eqs. $(10,11)$ can be estimated as a function of image speed on the focal plane $\left(V_{i}\right)$ as follows [10]:

$$
t_{\text {int }}=\frac{\Delta}{V_{i}}
$$

Any mismatch between the image speed on focal plane (target scanning rate) with the accumulated charge transfer frequency along TDI lines (electronic line reading frequency) will cause a longitudinal smear. Therefore, the image speed on focal plane must be estimated accurately and can be defined as:

$$
V_{i}=\frac{V_{s}}{D} f
$$

where $D$ is the slant distance between the satellite and target and $V_{s}$ is the resultant speed of sub-satellite point. However, the slant distance is expressed by [11]:

$$
D=\sqrt{\left(R_{E}+H\right)^{2}-R_{E}{ }^{2} \cos ^{2}(\alpha)}-R_{E} \sin (\alpha)
$$

where $R_{E}$ is the Earth radius, $H$ is the orbit altitude, and $\alpha$ is the satellite elevation angle as defined in Fig 5. Recall Eq. (13), $V_{s}$ is calculated by:

$$
V_{S}=\sqrt{V_{o}^{2}+V_{E}^{2}-2 V_{o} V_{E} \cos (i) \cos (\Phi)}
$$

where $V_{E}$ is the speed of the sub-satellite point due to Earth rotation around its axis, $i$ is the orbit inclination, $\Phi$ is the target latitude on the ground and $V_{o}$ is the speed of the sub-satellite point on the Earth due to satellite orbiting and defined by [10]:

$$
V_{o}=R_{E} \frac{\sqrt{\mu_{o}}}{\left(R_{E}+H\right)^{3 / 2}}
$$

where $\mu_{0}$ is the earth gravitational constant. Clearly, the more accurate is estimated the image speed on focal plane, the less the mismatch, the less the smear occurred. The accurate estimation of image speed depends on the exact values of $s, H, i$, and $\Phi$. In this analysis, two scenarios are assumed, the first scenario is the maximum illumination condition where sun zenith angle $\dot{\eta}=0^{\circ}$, satellite zenith angle $\alpha=0^{\circ}$ and the target latitude located on the equator. Consequently, these conditions correspond to the maximum exposure that can be registered by the sensor. The second scenario is the minimum illumination condition in which $\dot{\eta}=75^{\circ}, \alpha^{\prime}=40^{\circ}$ and target located on near 
polar latitude $\left(70^{\circ}\right)$. The results are shown in Table 2.

Table 2: Estimated integration time and line frequency

\begin{tabular}{|c|c|c|}
\hline Imaging scenario & Integration time for $N_{T D I}=1$ & Line frequency \\
\hline $\begin{array}{c}\text { Max illumination condition } \\
\left(\dot{\eta}=0^{\circ}, \dot{\alpha}=0^{\circ}, \Phi \text { is located on }\right. \\
\text { the equator })\end{array}$ & $0.035 \mathrm{~ms}$ & 28565 line $/ \mathrm{s}$ \\
\hline $\begin{array}{c}\text { Min illumination condition } \\
\left(\dot{\eta}=75^{\circ}, \quad \alpha=40^{\circ}, \Phi \text { is located }\right. \\
\text { near the poles })\end{array}$ & $0.045 \mathrm{~ms}$ & 22210 line $/ \mathrm{s}$ \\
\hline
\end{tabular}

In order to achieve a good image quality, the absolute SNR shall be more than 100 [12]. By using Eqs. (7, 10 and 11), the resulted absolute SNR at different target albedos and TDI lines for the both predetermined scenarios are depicted in Fig. 2 and 3, respectively. It is noticed that in the case of max illumination scenario, a saturation occurs (signal exceeds the pixel $F W C$ ) only when selecting $N_{T D I}=128$ lines at albedos higher than 0.5. By decreasing the number of TDI lines one step down to 64 TDI lines, no saturation occurs. In the case of minimum illumination condition, no saturation occurs at 128 TDI lines, and the SNR reaches to 100 at albedos $0.13,0.225,0.375$, 0.66 using 128, 64, 32, 16 TDI lines consequently. Accordingly, the choice of 128 TDI lines will be excluded because of the saturation at maximum illumination scenario. Furthermore, the selection of 8 TDI lines is not preferable to be used in the case of minimum illumination scenario as the SNR $<100$ for all albedos. Thus, the selection of the number of TDI lines depends on the imaging illumination scenario and the average albedo of the scene.

If it is required to define the optimum number of TDI lines in any imaging scenario, it will be defined according to the following criteria: the highest number of TDI lines at which no saturation occurs in the case of maximum illumination condition and, at the same time, the SNR higher than 100 for the case of minimum illumination scenario. The optimum number of TDI lines satisfied the criteria was $N_{T D I}=64$ TDI lines because of firstly at maximum illumination scenario, no saturation occurs, Secondly the SNR higher than 100 starting from albedo 0.1 at maximum illumination and starting from albedo 0.225 in minimum illumination which is satisfactory.

\subsection{Differential SNR}

The differential SNR $\left(S N R_{\triangle \rho}\right)$ is defined by considering the target under observation as the difference in reflectance between two adjacent targets [8]:

$$
S N R_{\Delta \rho}=\frac{S_{\text {tar } \mid \rho_{\text {high }}-}-S_{\text {tar } \mid} \rho_{\text {low }}}{\sigma_{\text {tot } \mid \rho_{\text {high }}}}=\frac{S_{\text {tar } \mid \Delta \rho}}{\sigma_{\text {tot } \mid \rho_{\text {high }}}}
$$

where $\rho_{\text {high }}$ and $\rho_{\text {low }}$ are the higher and lower albedos of the neighboring targets respectively. Taking the same criteria presented in [2], the differential SNR at Nyquist frequency $\left(S N R_{\Delta \rho} \times M T F_{\text {Nyquist }}\right)$ should be more than 2.8 and the differential SNR is 
around 14 for albedos difference of $7 \%$ and $13 \%$ (i.e., $\Delta \rho=0.06$ ) for $N_{T D I}=8$ and sun elevation angle $30^{\circ}$. In the following assessment, the differential SNR is analyzed between two adjacent targets, the low albedo target is $7 \%$ and the high albedo target ranging ranges from $12 \%$ till $17 \%$ using 8 TDI lines at both predetermined scenarios as shown in Fig. 4. However, the results of the analysis are outlined in Table 3. It is clear that the both requirements are not satisfying for the minimum illumination conditions.

Table 3: $S N R_{\Delta \rho}$ analysis

\begin{tabular}{|c|c|c|c|c|c|c|}
\hline \multirow[t]{2}{*}{ Parameter } & \multicolumn{2}{|c|}{ Requirement } & \multicolumn{2}{|c|}{$\begin{array}{c}\text { Maximum } \\
\text { illumination } \\
\text { scenario }\end{array}$} & \multicolumn{2}{|c|}{$\begin{array}{c}\text { Minimum } \\
\text { illumination scenario }\end{array}$} \\
\hline & $\begin{array}{l}\text { Imaging } \\
\text { scenario }\end{array}$ & Value & $\begin{array}{l}\text { Imaging } \\
\text { scenario }\end{array}$ & Value & $\begin{array}{l}\text { Imaging } \\
\text { scenario }\end{array}$ & Value \\
\hline$S N R_{\Delta \rho}$ & \multirow{2}{*}{$\begin{array}{c}\alpha^{\prime}=0^{0} \\
\eta^{\prime}=60^{\circ} \\
N_{T D I}=8 \\
\Delta \rho=0.06\end{array}$} & $\sim 14$ & \multirow{2}{*}{$\begin{array}{c}\dot{\alpha}=0^{0} \\
\dot{\eta}=0^{0} \\
N_{T D I}=8 \\
\Delta \rho=0.06\end{array}$} & 14.02 & \multirow{2}{*}{$\begin{array}{l}\dot{\alpha}=40^{\circ} \\
\dot{\eta}=75^{0} \\
N_{T D I}=8 \\
\Delta \rho=0.06\end{array}$} & 7 \\
\hline$S N R_{\Delta \rho} \times M T F_{\text {Nyquist }}$ & & $>2.8$ & & 2.67 & & 1.33 \\
\hline
\end{tabular}

\section{Spatial resolution}

The spatial resolution of the electro-optical remote sensing satellites is considered the most significant performance metric. The spatial resolution can be either represented in terms of the pixel projection onto the ground according to the acquisition geometry and named by geometrical resolution $\left(G S D_{G M}\right)$, or in terms of the maximum resolvable spatial frequency detected by the imaging system $\left(v_{\max }\right)$ and, in this case, it is named the cutoff resolution $\left(R_{\text {cut }}\right)$ reflecting the actual system resolution [13]. In fact, the required geometrical resolution cannot be achieved by real systems due to degradation effects of optics, noise, atmosphere, smear, etc.

\subsection{Geometrical Resolution}

Following the traditional approach, most modern CCDs have equal pixel pitch in both $x$ and $y$ directions (i.e., $p=p_{x}=p_{y}$ ). Thus, the geometrical resolution in terms of ground sampling distance (GSD) at nadir can be defined by [11]:

$$
G S D_{x}=G S D_{y}=\frac{p H}{f}=\frac{H}{2 v_{n y q} f}
$$

where $x$ and $y$ refer to the across-scan and along-scan directions, respectively. Then, the geometric resolution is the geometric mean of both GSD components and is defined as:

$$
G S D_{G M}=\sqrt{G S D_{x} G S D_{y}}
$$


Fig. 5 shows the imaging geometry taking in consideration the earth curvature, the $G S D_{G M}$ at off-nadir angles considering earth curvature can be calculated by [9]

$$
G S D_{G M}=\frac{p D}{f \sqrt{\sin \alpha}}=\frac{D}{2 v_{n y q} f \sqrt{\sin \alpha}}
$$

$\alpha$ can be related to the $\theta$ through the relation [11]:

$$
\alpha=\arccos \left[\frac{R+H}{R} \sin \theta\right]
$$

The $G S D_{G M}$ has been calculated for the both max and min illumination scenarios and found to vary from $0.25 \mathrm{~m}$ at $\alpha=0^{\circ}$ to $0.36 \mathrm{~m}$ at $\alpha=40^{\circ}$.

\subsection{Cut-off Resolution}

The cut-off resolution ( $R_{c u t}$ ) is limited by the maximum resolvable spatial frequency, so, by substituting vnyq in Eq. (18) with $v_{\max }$, then, the cutoff resolution at nadir can be represented by [13]:

$$
R_{c u t}=\frac{H}{2 v_{\max } f}
$$

In case of off-nadir imaging, the geometric mean of the cutoff resolution $\left(R_{G M}\right)$ can be represented by:

$$
R_{G M}=\frac{D}{2 v_{\max } f \sqrt{\sin \alpha}}
$$

The MTF of an imaging system is defined as the spatial frequency response of the sine wave target. In fact, the sine wave targets are hard to manufacture, so, the square wave targets (bar targets) are usually used instead. The spatial frequency response of a bar target is called the contrast transfer function (CTF). For the same imaging system, the MTF and the CTF are not equal. However, using the bar targets for evaluation, it is required to convert the system MTF to its equivalent CTF. A relationship between them is defined by [14]:

$$
C T F=\frac{4}{\pi} M T F
$$

A standard tri-bar test pattern (or sometimes named by Mira target) is commonly utilized to evaluate the cut-off resolution of the imaging system. Detailed study on the analysis of the observer threshold signal-to-noise ratio (SNR thr) to the Mira test pattern image was performed so that [15]

$$
S N R_{t h r}(X)=3-70 X+901 X^{2}-5518 X^{3}+17411 X^{4}-27126 X^{5}+16711 X^{6}
$$

In fact, the differential SNR as a function of spatial frequency is defined by [13]:

$$
S N R_{\Delta \rho}(v)=S N R_{\Delta \rho}(v \rightarrow 0) \times M T F_{t o t}(v)
$$


Then, the maximum resolvable spatial frequency is the spatial frequency at which the system SNR intersects the threshold SNR so that

$$
S N R_{\Delta \rho}(v \rightarrow 0) \times \frac{4}{\pi} M T F_{t o t}(v)=S N R_{t h r}(v)
$$

Applying the previous procedures for the two scenarios, the graphical solutions show $R_{\text {cut }}$ equals $0.264 \mathrm{~m}$ and $0.43 \mathrm{~m}$ for the maximum and minimum illumination scenarios as shown in Fig. 6 and 7, respectively.

\section{Conclusion}

The performance analysis and evaluation of a very high-resolution telescope with a GSD of $0.25 \mathrm{~m}$ presented in [3] and suitable for the next-generation earth observation system has been investigated. The fundamental system metrics considering the MTF, absolute SNR, differential SNR, geometric and cut-off resolutions are analyzed. The assessment shows that, under minimum and maximum illumination conditions, the predictable performance of the proposed telescope under study gives promising results and is expected to give an acceptable image quality.

\section{Figures}

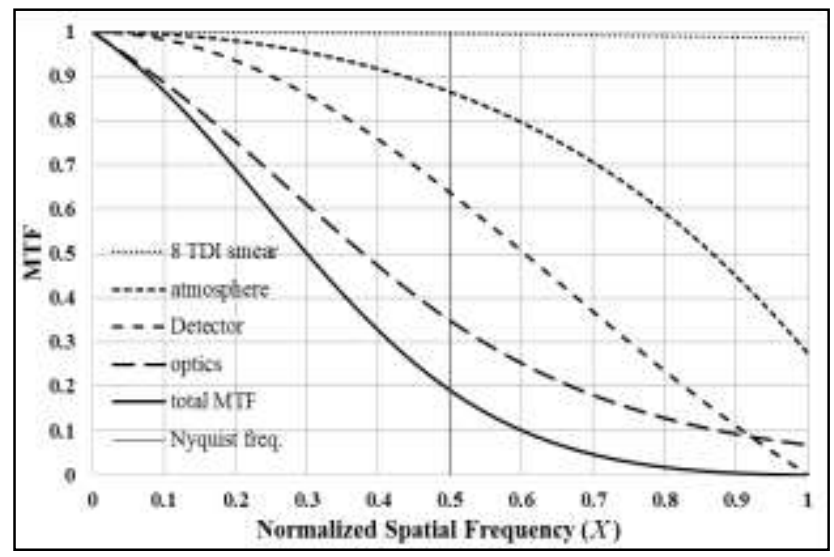

(a)

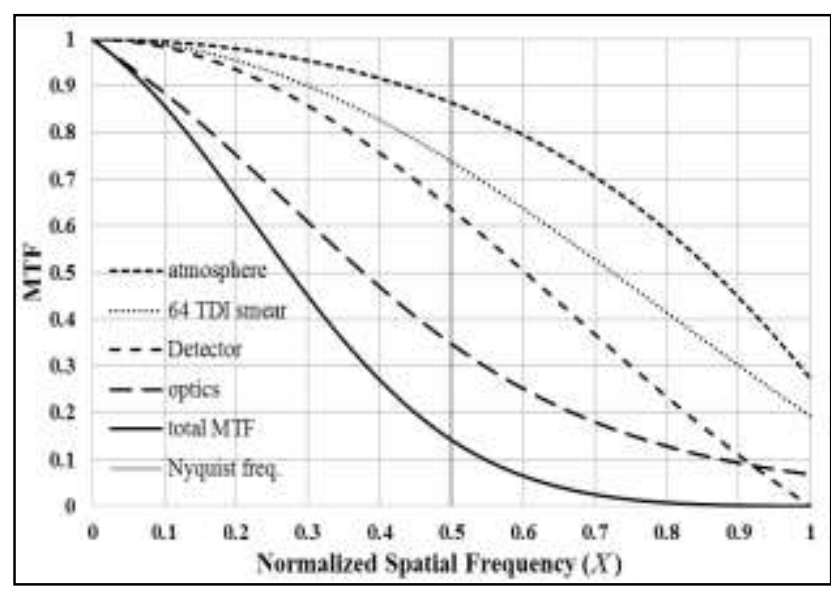

(b)

Fig. 1 Total MTF when a) $N_{T D I}=8$ and $\theta=0^{\circ} \quad$ b) $N_{T D I}=64$ and $\theta=36.5^{\circ}$ 


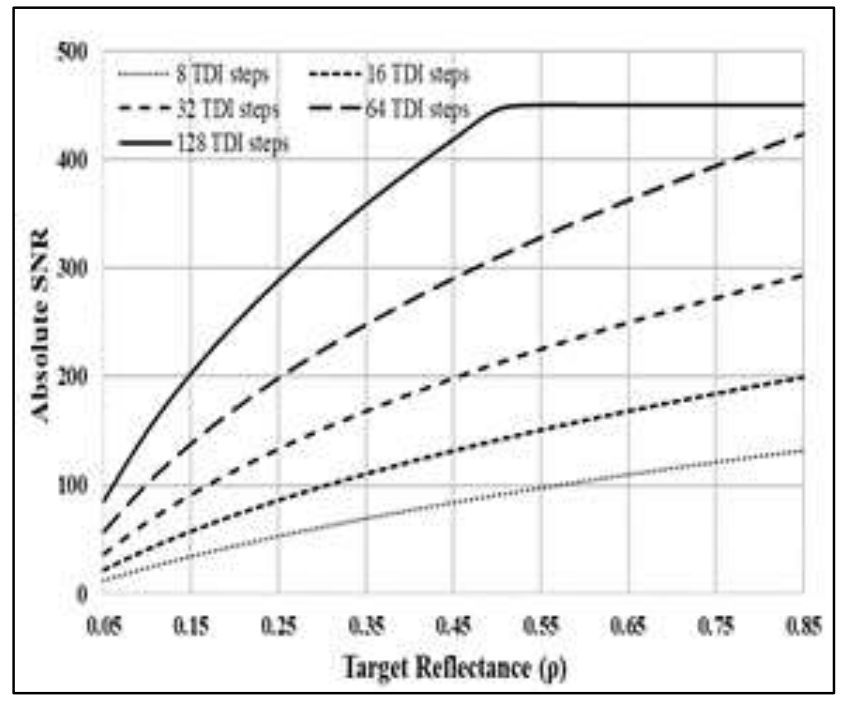

Fig. 2 Absolute SNR as a function of target reflectance at maximum illumination condition

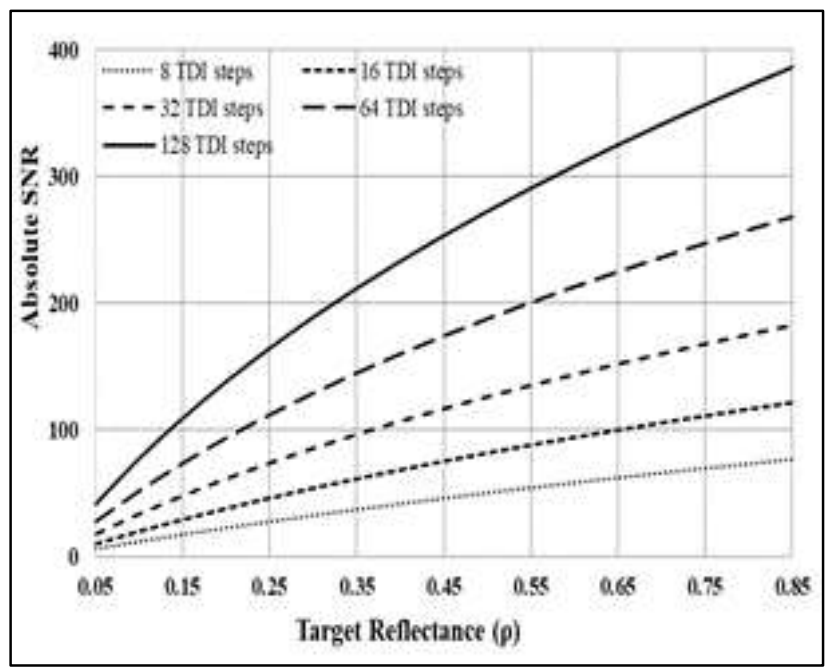

Fig. 3 Absolute SNR as a function of target reflectance at minimum illumination condition 


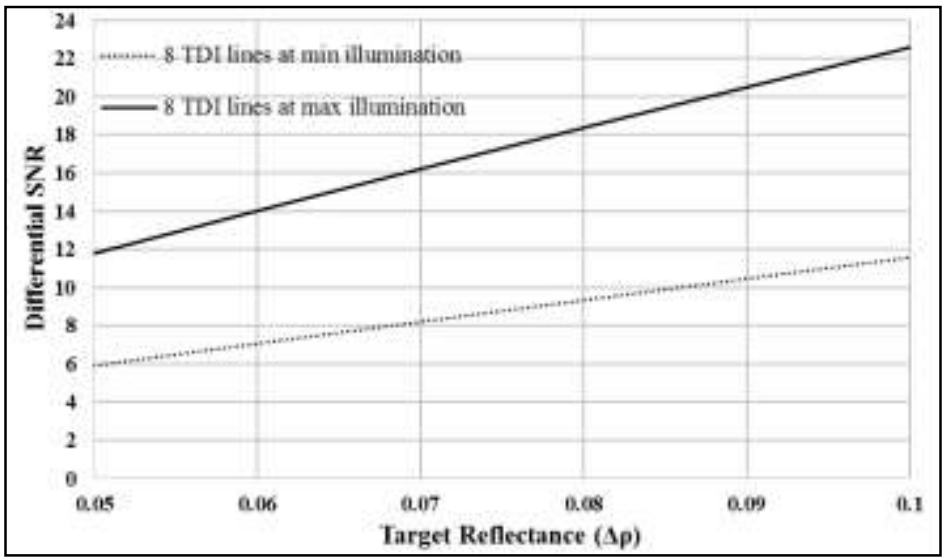

Fig. 4 Differential SNR as a function of target reflectance

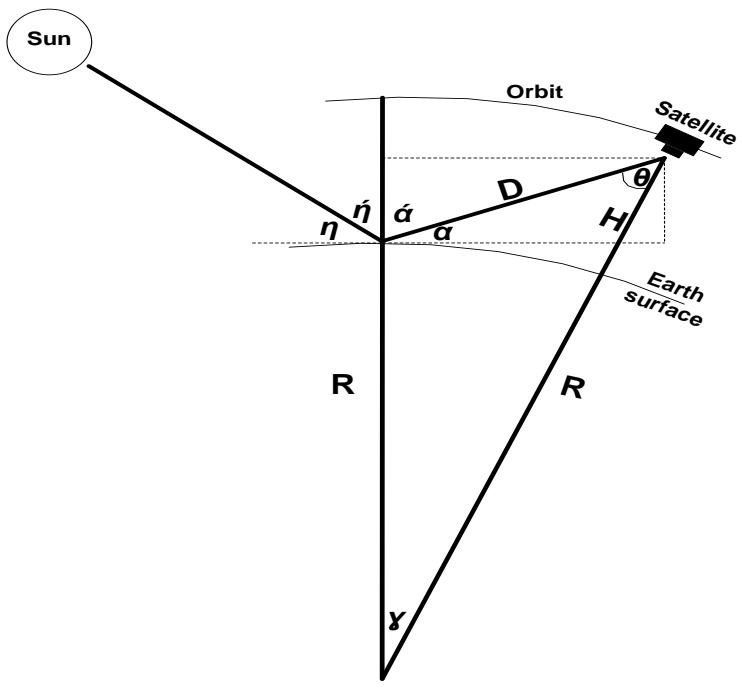

Fig. 5 Imaging geometry considering the earth curvature

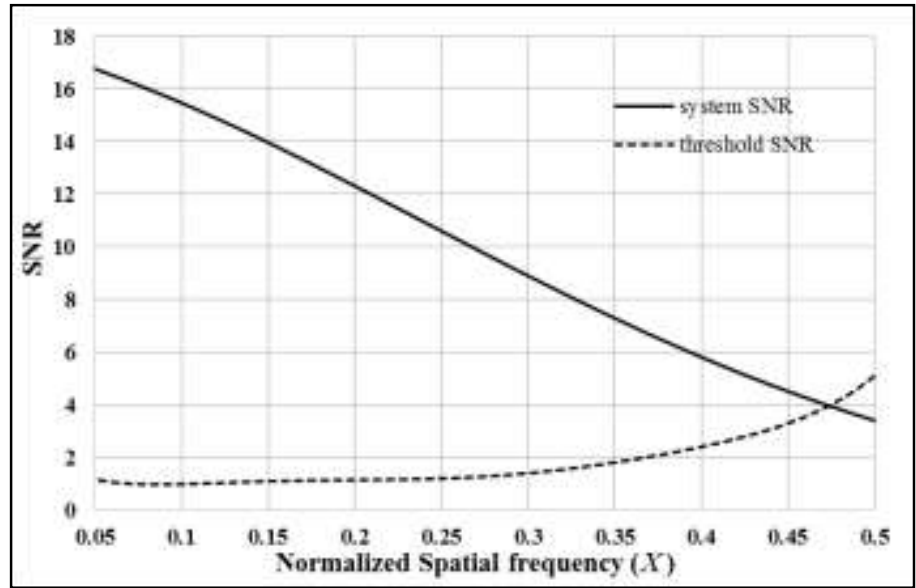

Fig. 6 The graphical solution of the maximum resolvable spatial frequency at $\alpha=$ $0^{\circ}, \dot{\eta}=0^{\circ}, 8$ TDI lines and $\Delta \rho=0.06$ 


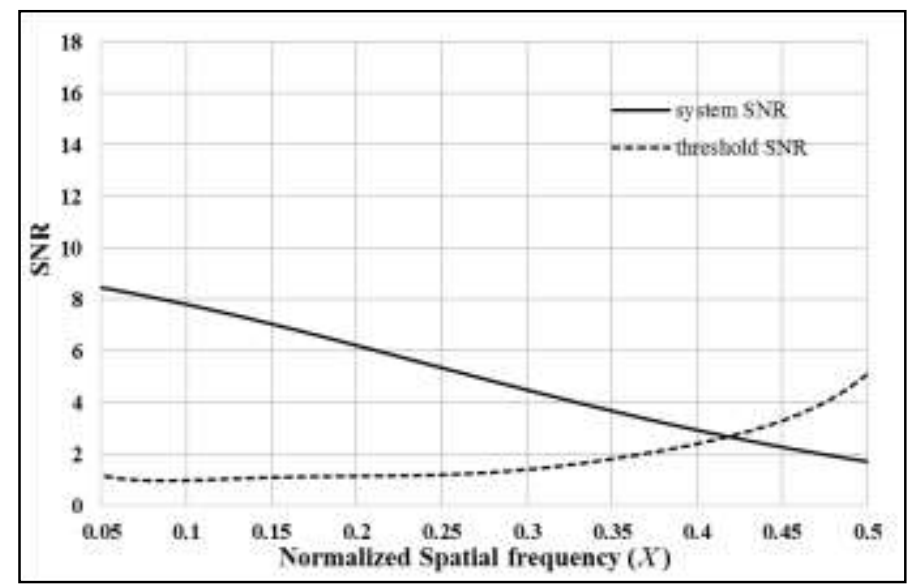

Fig. 7 The graphical solution of the max resolvable spatial frequency at $\alpha=40$, $\dot{\eta}=75^{\circ}, 8 \mathrm{TDI}$ lines and $\Delta \rho=0.06$

\section{Reference}

[1] X. Kuna, C. Shi-xiang and Y. Chun-yu, "Optimization design method of optical remote sensor based on imaging chain simulation," MATEC Web of Conferences 114, 04013, May 2017.

[2] T. Musabayev, M. Moldabekov, M. Nurguzhin, S. Dyussenev, S. Murushkin, B. Albazarov and V. Ten, "Earth observation system of the republic of Kazakhstan," International Astronautical Congress, IAC-13-B1.2.3, 2013.

[3] M. Metwally, Taher M. Bazan, F. Eltohamy, and M. Fayhy "Optical design, tolerance analysis, and baffling of very high-resolution satellite telescopes," accepted at ICEENG 2018.

[4] S. Wong and R. Jassemi-Zargani, "Predicting image quality of surveillance sensors," Defense Research and Development Canada, Scientific Report, Oct. 2014.

[5] K. Burshtynska and I. Dolynska "Space remote sensing systems transmission capabilities modeling," Geomatics, Land management and Landscape, No 3, pp. 27-35, 2013.

[6] Steven L. Smith, Robert D. Fiete and Theodore A. Tantalo "Understanding image quality losses due to smear in high-resolution remote sensing imaging systems," Optical Engineering, Vol. 38 No. 5, May 1999.

[7] Walid A. Wahballah, Taher M. Bazan, Fawzy El-Tohamy, and Mahmoud Fathy "Analysis of smear in high-resolution remote sensing satellites," Proc. of SPIE, Vol. $10000.100001 \mathrm{~J}-2,2016$.

[8] R. Fiete., and T. Theodore, "Comparison of SNR image quality metrics for remote sensing systems," Optical Engineering, vol. 40, pp. 574-585, April 2000.

[9] R. Auelmann, "Image Quality Metrics," at http://www.techarchive.org/wpcontent/themes/

boilerplate/largerdocs/Image\%20Quality\%20Metrics.pdf, last accessed in $1 / 3 / 2018$.

[10]I. Prudyus, V. Tkachenko, P. Kondratov, S. Fabirovskyy, L. Lazko, and A. Hryvachevskyi, "Factors affecting the quality of formation and resolution of images in remote sensing systems," Computational Problems of Electrical Engineering, vol. 5, pp. 41-46, 2015. 
[11] Robert D. Fiete, "Image quality and IFN/p for remote sensing systems," Optical Engineering, vol.38, pp. 1229-1240, July 1999.

[12] M. Hashim, W. Kadir and W. Hazli, "Radiometric and geometric information content of TiungSAT-1 MSEIS Data," In: TiungSAT-1: From Inception to Inauguration. Astronautic Technology, pp. 185-200. ISBN 983-867-193-2, 2001.

[13] Meira Citroen, Guy Raz and Michael Berger "Noise Equivalent Reflectance Difference (NERD) vs. Spatial Resolution (SR) as a good measure for system performances," Proc. of SPIE, vol. 7087 708705, pp. 1-11, 2008.

[14]N. Nill "Conversion between sine wave and square wave spatial frequency response of an imaging system," MITRE Corporation technical report, July 2001

[15]A. Sekoroski, "Method for estimating the frequency energetic characteristics threshold of optoelectronic path," in Russian, at http://www.elibrary.It/resursai/Uzsienio\%20leidi niai/MFTI/2003/112r.pdf, last accessed in 1/3/2018. 